\title{
BridgeDb app: unifying identifier mapping services for
}

\section{Cytoscape [version 1; peer review: 2 approved]}

\author{
Jianjiong Gao1 ${ }^{1}$ Chao Zhang ${ }^{2}$, Martijn van Iersel ${ }^{3}$, Li Zhang ${ }^{4}$, Dong Xu5 \\ Nikolaus Schultz'1,6, Alexander R. Pico (iD) 7
}

\footnotetext{
${ }^{1}$ Kravis Center for Molecular Oncology, Memorial Sloan Kettering Cancer Center, New York, NY 10065, USA

2Institute for Computational Biomedicine, Weill Cornell Medical College, New York, NY 10065, USA

${ }^{3}$ General Bioinformatics, Reading, Birkshire, RG4 7RT, UK

${ }^{4}$ School of Computer Science and Engineering, Changchun University of Technology, Changchun, 130012, China

${ }^{5}$ Department of Computer Science,C.S. Bond Life Sciences Center, University of Missouri, Columbia, MO 65211, USA

${ }^{6}$ Department of Epidemiology and Biostatistics, Memorial Sloan Kettering Cancer Center, New York, NY 10065, USA

${ }^{7}$ Gladstone Institutes, San Francisco, CA 94158, USA
}

\section{V1 First published: $01 \mathrm{Jul}$ 2014, 3:148 \\ https://doi.org/10.12688/f1000research.4521.1}

Latest published: 01 Jul 2014, 3:148

https://doi.org/10.12688/f1000research.4521.1

\section{Abstract}

The BridgeDb app for Cytoscape allows users to map and annotate identifiers of genes, proteins and metabolites in the context of biological networks. The app greatly simplifies the identifier mapping process in Cytoscape by providing a unified interface to different mapping resources and services. The app also provides a programming interface via Cytoscape Commands that can be utilized for identifier mapping by other Cytoscape apps. In this article we provide a technical guide to the BridgeDb app for mapping identifiers in Cytoscape.

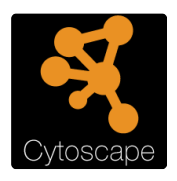

This article is included in the Cytoscape gateway.

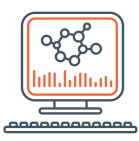

This article is included in the Bioinformatics

gateway.

\section{Open Peer Review \\ Approval Status \\ 1 \\ 2 \\ version 1 \\ 01 Jul 2014

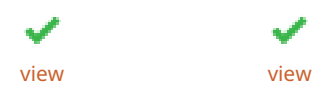 \\ 1. Benno Schwikowski, Institut Pasteur, Paris, \\ France \\ 2. Olivier Elemento, Weill Cornell Medical \\ College, New York, NY, USA \\ Any reports and responses or comments on the article can be found at the end of the article.}


Corresponding author: Jianjiong Gao (jianjiong.gao@gmail.com)

Competing interests: No competing interests were disclosed.

Grant information: This work was partially funded by the Google Summer of Code program (JG, CZ, and LZ), the Robertson Foundation (NS), and by NIGMS R01-GM100039 (ARP).

The funders had no role in study design, data collection and analysis, decision to publish, or preparation of the manuscript.

Copyright: $\odot 2014$ Gao J et al. This is an open access article distributed under the terms of the Creative Commons Attribution License, which permits unrestricted use, distribution, and reproduction in any medium, provided the original work is properly cited. Data associated with the article are available under the terms of the Creative Commons Zero "No rights reserved" data waiver (CC0 1.0 Public domain dedication).

How to cite this article: Gao J, Zhang C, van Iersel $\mathrm{M}$ et al. BridgeDb app: unifying identifier mapping services for Cytoscape [version 1; peer review: 2 approved] F1000Research 2014, 3:148 https://doi.org/10.12688/f1000research.4521.1

First published: 01 Jul 2014, 3:148 https://doi.org/10.12688/f1000research.4521.1 


\section{Introduction}

Cytoscape ${ }^{1}$ is a powerful network visualization tool and platform for data integration and analysis. However, identifier mapping remains a challenge when working with biological data from different sources. We developed the Bridge $\mathrm{Db}$ app for Cytoscape to provide utilities for mapping and annotating identifiers in the network context. Built on the BridgeDb open-source framework for identifier mapping ${ }^{2}$, the BridgeDb app provides a graphical user interface (GUI) for users and a command interface for other Cytoscape apps to perform identifier mapping in Cytoscape networks. As a result, BridgeDb enables or simplifies the processes of identifier translation, biological entity unification, and functional annotation.

\section{Implementation}

The BridgeDb identifier mapping framework (http://bridgedb.org/) ${ }^{2}$ was designed to provide standardized access to gene, protein and metabolite identifier mapping services such as Ensembl BioMart ${ }^{3}$, Synergizer ${ }^{4}, \mathrm{PICR}^{5}$ and BridgeDb web services and BridgeDb database files. By creating a Java-based abstract layer, BridgeDb enables bioinformatics applications to connect to different mapping resources through the same interface, which greatly alleviates the burden of exploring, maintaining and switching between resources.

Built upon BridgeDb framework and API, the BridgeDb app for Cytoscape can be used to connect to different mapping resources and map identifiers in Cytoscape netwoks. The BridgeDb app was implemented based on the Cytoscape 3 API. Its predecessor was the CyThesarus plugin for Cytoscape 2. To take advantage of the new OSGi based architecture in Cytoscape 3 and its clearly defined and simplified API, we have rewritten the CyThesaurus plugin into an OSGi bundle app. Figure 1 illustrates the implementation details. The identifier mapping API of BridgeDb framework was wrapped by Cytoscape Task and TaskFactory API, which provide identifier mapping utilities to users through graphical user interfaces for managing mapping resources and performing identifier mapping. The BridgeDb app Tasks were also registered to Cytoscape as command services allowing other apps, such as Mosaic ${ }^{6}$ and $\mathrm{NOA}^{7}$ apps, and the Merge Network tool, to take advantage of BridgeDb app's identifier mapping capacities.

\section{Results}

\section{BridgeDb app user interface}

The BridgeDb app provides an intuitive GUI to perform identifier mapping in Cytoscape networks, consisting of two interactive dialogs for resource management and identifier mapping, respectively, which can be opened via the menu item Apps/BridgeDb.

The resource management dialog allows user to add, remove, and select or deselect mapping resources. Once the resources are configured, they will be saved in a global property file and therefore shared among different Cytoscape sessions. Currently BridgeDb app supports local and remote delimited text files, BridgeDb database files, BridgeDb web service, BioMart web service, and Synergizer web service. Web services are easy to access and up to date and therefore are preferable for annotating small to medium-size networks (less than 1,000 nodes). It is highly recommended to download and use the BridgeDb database files for mapping identifiers in large networks in order to save time. Local delimited text files

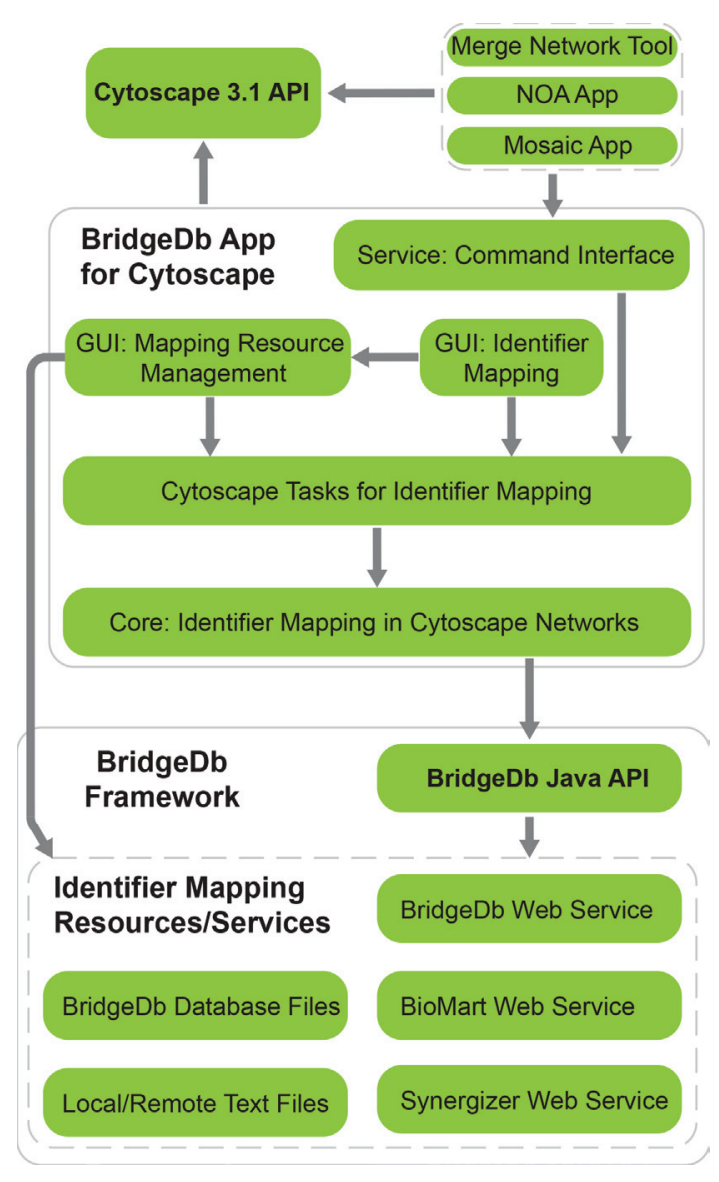

Figure 1. BridgeDb app implementation.

are useful when mapping between non-standard or customized identifiers. To avoid ambiguity, it is recommended to select only one resource unless multiple resources are believed to be complementary to each other. Particularly, resources for different species should not be selected at the same time. Database or text files are also preferable when reproducibility is essential. We will update the app to support more mapping resources as BridgeDb API keeps being developed.

The identifier mapping dialog is the main interface for mapping identifiers stored in the node table in the selected Cytoscape network. The user needs to choose the source identifier types, columns in the node table that contain the source identifiers, target identifier types, and columns to save the target identifiers. When mapping, all specified source identifier types in all selected resources will be queried for matching identifiers. If one source identifier can be mapped onto multiple target identifiers, all target identifiers can be saved as a list in the node table. If a target column exists in the node table, all values in the column will be overwritten with the target identifiers; otherwise, a new column will be created and filled with the target identifiers. Besides a set of supported identifier types (e.g., Entrez Gene ID and UniProt accession), a mapping resource such as BridgeDb database may also have a set of supported attributes (e.g., gene symbol and description). 
BridgeDb app command interface

The BridgeDb app also provides a set of Cytoscape commands, which can be used by scripting or by other Cytoscape apps (client apps) to take advantage of the identifier mapping capacities provided by BridgeDb app. Table 1 provides a selected list of commands supported by BridgeDb. Every command has an optional argument 'appName' in order to allow every client app to have its own set of identifier mapping resources. Morris et al. (setsApp ${ }^{8}$, published together in the Cytoscape App collection ${ }^{9}$ ) provided an example of how to use commands in client apps.

\section{Example use cases}

Use cases of Bridge Db app include identifier translation, biological entity unification, and functional annotation in Cytoscape networks. Box 1 provides an example of how to use BridgeDb app to facilitate annotation and integration of networks from public databases. In particular, this example shows the steps to generate a TP53 interaction network by merging networks from different sources using Bridge Db app and Merge Network tool. Figure 2 illustrates the process of the Cytoscape app $\mathrm{NOA}^{7}$ annotating Cytoscape networks with Gene Ontology by utilizing BridgeDb app's command services.

Table 1. Selected BridgeDb app commands. Arguments with asterisks $\left({ }^{*}\right)$ are required.

\begin{tabular}{|c|c|c|}
\hline Command & Arguments & Description \\
\hline bridgedb resource add & $\begin{array}{l}\text { classPath }=\text { IDMapper class path } \\
\text { connString=BridgeDb connection string } \\
\text { displayName = display name } \\
\text { appName=name of client Cytoscape app }\end{array}$ & $\begin{array}{l}\text { Add a mapping resource. appName is the } \\
\text { name of the client Cytoscape app that is } \\
\text { using this command. }\end{array}$ \\
\hline bridgedb resource remove & $\begin{array}{l}\text { connString=BridgeDb connection string } \\
\text { appName }=\text { name of Cytoscape app }\end{array}$ & Remove a mapping resource. \\
\hline bridgedb resource select & $\begin{array}{l}\text { connString }=\text { BridgeDb connection string } \\
\text { select=true or false } \\
\text { appName=name of client Cytoscape app }\end{array}$ & Select or deselect an mapping resource. \\
\hline bridgedb id mapping & $\begin{array}{l}\text { network=Cytoscape network name* } \\
\text { sourceColumn=source column in node table* } \\
\text { targetColumn=source ID type } \\
\text { sourceldType }=\text { target column in node table } \\
\text { targetldType }=\text { target ID type } \\
\text { appName }=\text { name of client Cytoscape app }\end{array}$ & $\begin{array}{l}\text { Map identifiers from a column in node } \\
\text { table to another in a Cytoscape network. }\end{array}$ \\
\hline $\begin{array}{l}\text { bridgedb resource config } \\
\text { dialog }\end{array}$ & appName=name of client Cytoscape app & $\begin{array}{l}\text { Open the resource management/ } \\
\text { configuration dialog. }\end{array}$ \\
\hline bridgedb main dialog & appName =name of client Cytoscape app & Open the main BridgeDb app dialog. \\
\hline
\end{tabular}

Box 1. Example: Using BridgeDb app for merging TP53 interaction networks from different sources

1. Install and start Cytoscape 3.1.0 or above

2. Select File $>$ Import $>$ Network $>$ Public Databases. .

3. Enter search condition TP53 AND human, press Search, select database IntAct, and click Import

4. After importing, click No when asking about whether to manually merge networks, and close the import dialog

5. If needed, install BridgeDb from Apps $>$ App Manager

6. Select File $>$ Import $>$ Network $>$ URL. . . Example:Human Protein-Protein: Rual et al. . . . Nature 2005

7. Use default when asking about setting of the new network

8. Select Apps $>$ BridgeDb $>$ Manager ID Mapping Resources

9. Click Databases, Select database type .bridge

10. Click Download, download Hs_Derby_[date].zip, and unzip the file

11. In Cytoscape, select the unzipped .bridge file

12. Review supported identifier types and click Close

13. Select network rual.sif in Network panel
14. Select Apps $>$ Bridge $D b>$ Map Identifiers

15. Select name as the Source Column in Node Table

16. Select Entrez Gene as Source ID Type(s)

17. Select Uniprot/TrEMBL as Target ID Type

18. Click Insert in the destination ID types table

19. Select Attribute: Symbol as the second Target ID Type

20. Click $\boldsymbol{O K}$, wait, then click No to close

21. Search for TP53 in the Cytoscape search box

22. Select Select $>$ Nodes $>$ First Neighbors of Selected Nodes $>$ Undirected

23. Select File $>$ New $>$ Network $>$ From selected nodes, all edges

24. Select Tool $>$ Merge $>$ Networks. . .

25. Select the network from IntAct and rual.sif(1)

26. Click Advanced Network Merge

27. Select Matching columns: uniprotkb accession for IntAct, and Uniprot/TrEMBL for rual.sif(1)

28. Click Merge to get a TP53 interaction network merged from the two sources 


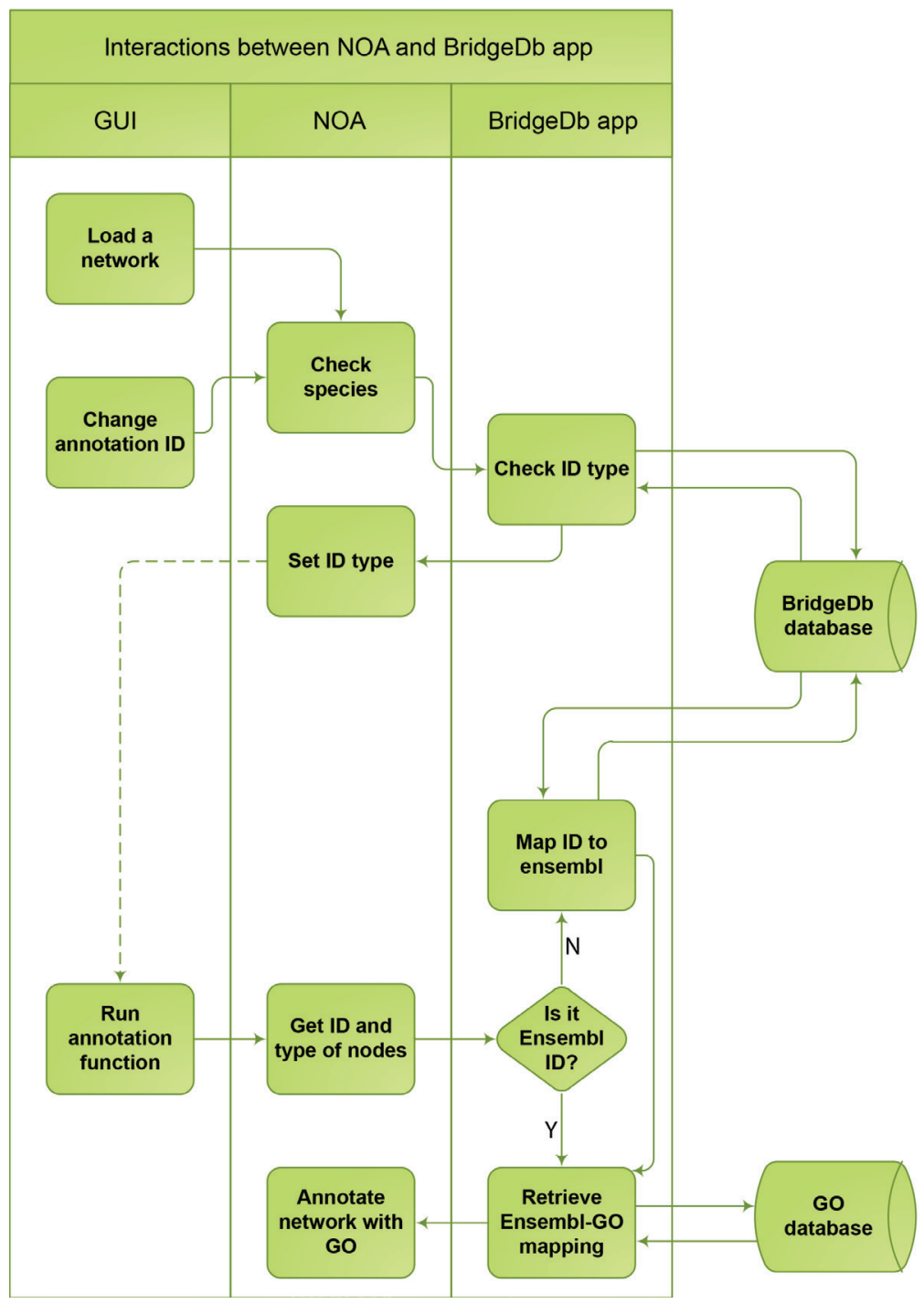

Figure 2. NOA app utilizing BridgeDb app services for gene ontology annotation.

\section{Conclusions}

In this paper, we presented the BridgeDb app for Cytoscape. By providing a unified interface to various mapping resources, BridgeDb app enables identifier mapping in the network context and therefore greatly eases the process of data integration in Cytoscape.

\section{Software availability}

Software available from: http://apps.cytoscape.org/apps/bridgedb

Latest source code: https://github.com/jjgao/bridgedb.cytoscape

Source code as at the time of publication: https://github.com/F1000Research/bridgedb.cytoscape
Archived source code as at the time of publication: http://www. dx.doi.org/10.5281/zenodo. $10465^{10}$

License: Lesser GNU Public License 2.1: https://www.gnu.org/licenses/old-licenses/lgpl-2.1.html

Author contributions

JG, CZ, MI and ARP participated in the design of the described software. JG and MI participated in the development of Bridge $\mathrm{Db}$ framework. JG, CZ and LZ implemented the BridgeDb app. JG, CZ, MI, DX, NS and ARP contributed to the development and writing of this article. 
Competing interests

No competing interests were disclosed.

Grant information

This work was partially funded by the Google Summer of Code program (JG, CZ, and LZ), the Robertson Foundation (NS), and by NIGMS R01-GM100039 (ARP).
The funders had no role in study design, data collection and analysis, decision to publish, or preparation of the manuscript.

\section{Acknowledgements}

We thank Scooter Morris for technical support on command interface, Kristina Hanspers for beta testing, and Gary Bader, Mike Smoot, Keiichiro Ono and David States for providing useful comments.

References

1. Shannon P, Markiel A, Ozier O, et al:: Cytoscape: a software environment for integrated models of biomolecular interaction networks. Genome Res. 2003 13(11): 2498-2504.

PubMed Abstract | Publisher Full Text | Free Full Text

2. van lersel MP, Pico AR, Kelder T, et al:: The BridgeDb framework: standardized access to gene, protein and metabolite identifier mapping services. $B M C$ bioinformatics. 2010; 11(1): 5 .

PubMed Abstract | Publisher Full Text | Free Full Text

3. Kasprzyk A, Keefe D, Smedley D, et al.: EnsMart: a generic system for fast and flexible access to biological data. Genome Res. 2004; 14(1): 160-169. PubMed Abstract | Publisher Full Text | Free Full Text

4. Berriz GF, Roth FP: The Synergizer service for translating gene, protein and other biological identifiers. Bioinformatics. 2008; 24(19): 2272-2273. PubMed Abstract | Publisher Full Text | Free Full Text

5. Côté RG, Jones $\mathrm{P}$, Martens L, et al.: The Protein Identifier Cross-Referencing
(PICR) service: reconciling protein identifiers across multiple source databases. BMC bioinformatics. 2007; 8(1): 401 PubMed Abstract | Publisher Full Text | Free Full Text

6. Zhang C, Hanspers K, Kuchinsky A, et al:: Mosaic: making biological sense of complex networks. Bioinformatics. 2012; 28(14): 1943-1944. PubMed Abstract | Publisher Full Text | Free Full Text

7. Zhang $\mathrm{C}$, Wang J, Hanspers $\mathrm{K}$, et al:: NOA: a cytoscape plugin for network ontology analysis. Bioinformatics. 2013; 29(16): 2066-2067.

PubMed Abstract | Publisher Full Text | Free Full Text

8. Morris JH, Wu A, Doncheva NT, et al:: setsapp: Set operations for cytoscape nodes and edges. Manuscript submitted for publication. F1000Research. 2014

9. The cytoscape app collection. F1000Research. 2014.

10. Gao J, Zhang C, van lersel M, et al:: F1000Research/bridgedb.cytoscape. ZENODO. 2014

Data Source 


\section{Open Peer Review}

\section{Current Peer Review Status:}

\section{Version 1}

Reviewer Report 22 July 2014

https://doi.org/10.5256/f1000research.4835.r5522

(C) 2014 Elemento O. This is an open access peer review report distributed under the terms of the Creative Commons Attribution License, which permits unrestricted use, distribution, and reproduction in any medium, provided the original work is properly cited.

\section{Olivier Elemento \\ Institute for Computational Biomedicine, Weill Cornell Medical College, New York, NY, USA \\ The paper by Gao et. al. describes a Cytoscape app/plugin for cross-database mapping of gene, protein and metabolite identifiers in biological networks. For example, the app can map EntrezGene identifiers to UniProt identifiers for all genes or selected genes in a Cytoscape network. This is an important and useful application that among other application enables the merging of networks obtained from different sources (as shown in demonstration). The app is reasonably user-friendly and intuitive. Most importantly, it does not require any programming knowledge and is accessible via a GUI. I appreciate that it either uses local downloaded files for mapping (for heavy usage) or BridgeDb web services (for smaller networks). The demo in the paper worked flawlessly on my MacBook Air.}

Potential future improvements may include ortholog mapping e.g. from mouse to human to enable cross-species network analyses.

Competing Interests: No competing interests were disclosed.

I confirm that I have read this submission and believe that I have an appropriate level of expertise to confirm that it is of an acceptable scientific standard.

Reviewer Report 10 July 2014

https://doi.org/10.5256/f1000research.4835.r5304 
(C) 2014 Schwikowski B. This is an open access peer review report distributed under the terms of the Creative Commons Attribution License, which permits unrestricted use, distribution, and reproduction in any medium, provided the original work is properly cited.

\section{Benno Schwikowski}

Systems Biology Lab, Institut Pasteur, Paris, France

BridgeDB app provides Cytoscape users and app developers with powerful functionality to address the ID mapping problem in integrative data analysis. App user and command interfaces are well-documented; the workflow is functional and recommended for anyone wishing to learn how to use the app.

As a minor suggestion, it would be reassuring to new users if they were able to validate the result of the workflow example in some way.

This is a very useful extension of Cytoscape functionality with broad appeal.

Competing Interests: Our team is co-developer of Cytoscape.

I confirm that I have read this submission and believe that I have an appropriate level of expertise to confirm that it is of an acceptable scientific standard.

The benefits of publishing with F1000Research:

- Your article is published within days, with no editorial bias

- You can publish traditional articles, null/negative results, case reports, data notes and more

- The peer review process is transparent and collaborative

- Your article is indexed in PubMed after passing peer review

- Dedicated customer support at every stage

For pre-submission enquiries, contact research@f1000.com 